\title{
Conventional versus frozen elephant trunk for complex aortic arch pathology: What should we be doing now?
}

Wilson Y. Szeto, MD

See related article on pages 1286-93.

The management of aortic arch pathologies with extension into the distal aorta has remained a challenging clinical scenario. In their article in this issue of the Journal, Shrestha and colleagues ${ }^{1}$ from Hannover have demonstrated the evolution of the management of complex aortic arch pathologies in their institution during the last decade, progressing from the traditional elephant trunk to the frozen elephant trunk (FET). Their excellent results and their innovative vision should be recognized. Such refinement and advancement in the surgical management of complex arch pathologies have been similarly experienced and reported by other aortic centers of excellence. ${ }^{2,3}$ Although this evolution has resulted in improving results in both mortality and stroke during the last decade, the article of Shrestha and colleagues confirms the continued debate and controversies that remain and highlights further work and investigation that are required.

Throughout medical history, the introduction of new technology has improved the outcomes of patient care, and it is no different with stent-graft technology and aortic surgery. The introduction of the FET has undoubtedly added a valuable tool for the aortic surgeon in the treatment of aortic disease. Conceptually, total arch reconstruction can now be performed with lessened technical complexities and demands. Although the elephant trunk maybe "freezing" at Hannover, however, I believe in fact that their experience actually supports a more sophisticated approach to the management of arch pathologies that is anatomically and disease specific. In another words, not all arch pathologies are the same and therefore should not be treated the same with a ubiquitous procedure; that is, the FET for all.

At first glance, the mortality difference between the 2 techniques appears to be staggeringly in favor of the FET. On further inspection (and as even admitted by Shrestha

From the Division of Cardiovascular Surgery, University of Pennsylvania Medical Center, Penn Presbyterian Medical Center, Philadelphia, Pa.

Disclosures: Author has nothing to disclose with regard to commercial support.

Received for publication Feb 26, 2015; accepted for publication Feb 27, 2015; available ahead of print March 24, 2015.

Address for reprints: Wilson Y. Szeto, MD, Division of Cardiovascular Surgery, University of Pennsylvania Medical Center, Penn Presbyterian Medical Center, $51 \mathrm{~N}$ 39th St, Heart Vascular Pavilion Suite 2A, Philadelphia, PA 19104 (E-mail: Wilson.szeto@uphs.upenn.edu).

J Thorac Cardiovasc Surg 2015;149:1294-5

$0022-5223 / \$ 36.00$

Copyright (C) 2015 by The American Association for Thoracic Surgery

http://dx.doi.org/10.1016/j.jtcvs.2015.02.059

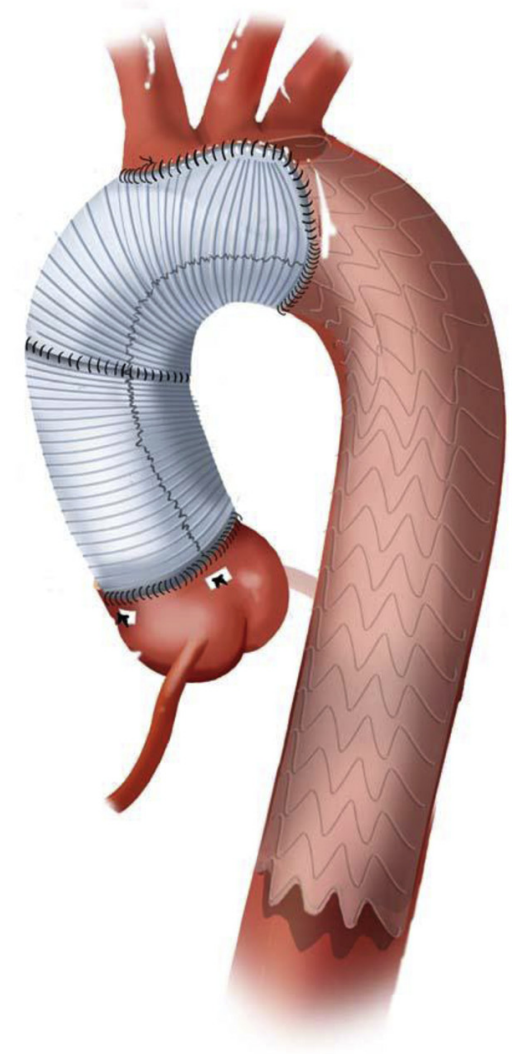

FIGURE 1. The frozen elephant trunk for acute proximal dissection.

and colleagues), however, the mortality difference in favor of the FET can be seen to be primarily driven by the higher proportion of acute dissection in the traditional elephant trunk group. Most centers would agree that in the setting of acute dissection, a traditional total arch reconstruction with traditional elephant trunk has been demonstrated in multiple series to be associated with high morbidity and mortality. Fundamentally, the FET allows the aortic surgeon to perform a "smaller" operation with lower morbidity and mortality (Figure 1). This is particularly important for patients with proximal aortic dissection with the primary tear site in the arch. Perhaps the FET may have its highest impact in this specific patient population, and it has proved to be an important surgical alternative.

For other aortic arch pathologies, however, the debate remains. In the setting of aneurysmal disease, the data are not as clear. In fact, this series, along with others, shows no differences in both short-term and midterm results. Interestingly, the distal reintervention rates are similar for the 
traditional elephant trunk and the FET. For chronic dissection, the differences remain just as unclear. Whether the FET influences and impacts the chronic dissection flap and offers better outcome with distal aortic remodeling remains to be determined. As stated by Shrestha and colleagues, this particular series does not have a robust number of chronic dissections treated with the traditional elephant trunk to make a valid comparison. Aortic type B dissection, or distal aortic dissection, also remains an interesting entity that requires further investigation. Retrograde type A aortic dissection is a dreaded complication associated with the treatment of type B dissection with thoracic endovascular aneurysm repair. Although the FET offers a conceptually sound therapeutic strategy, the notion of performing a total arch reconstruction with the FET in the setting of distal dissection is a major undertaking with an aggressive indication. Finally, patients with connective tissue disorders remain a controversial population of patients to treat with the FET. Distal aortic injury and progressive aortic dilatation continue to be major issues in this patient population, and they require further study.

In conclusion, both the traditional elephant trunk and the FET remain important therapeutic options in the management of aortic arch pathologies. The FET undoubtedly has proved to be an advance in surgical technique and provides an important therapeutic alternative in the management of complex aortic arch pathologies; however, it requires further investigation in determining its best utility and benefit. Before widespread clinically adaptation of the FET technology can be justified, further investigations to identifying the appropriate patients with the appropriate anatomic criteria will be needed. Particularly in the United States, where this technology is not currently commercially available, the FET concept has only been evaluated with the creative adaptation of thoracic endovascular aneurysm repair technology not designed specifically for this use. Larger studies with longer follow-up will be needed before more definitive conclusions can be made.

\section{References}

1. Shrestha M, Beckmann E, Krueger H, Fleissner F, Kaufeld T, Koigeldiyev N, et al The elephant trunk is freezing: The Hannover experience. $J$ Thorac Cardiovasc Surg. 2015;149:1286-93.

2. Vallabhajosyula P, Szeto WY, Pulsipher A, Desai N, Menon R, Moeller P, et al. Antegrade thoracic stent grafting during repair of acute DeBakey type I dissection promotes distal aortic remodeling and reduces late open distal reoperation rate. $J$ Thorac Cardiovasc Surg. 2014;147:942-8.

3. Di Bartolomeo R, Di Marco L, Cefarelli M, Leone A, Pantaleo A, Di Eusanio M, et al. The Bologna experience with the Thoraflex hybrid frozen elephant trunk device. Future Cardiol. 2015;11:39-43. 